\title{
Susceptibility Profile of Drug-Resistant Streptococcus pneumoniae Based on ELR
}

\author{
Avi Raju*, Eunice R. Santos, Eric V. Bakota, Biru Yang and Raouf R. Arafat \\ Houston Health Department, Houston, TX, USA
}

\section{Objective}

Our objective is to report the antimicrobial susceptibilities of Streptococcus pneumoniae received from a local safety net hospital via electronic laboratory reporting (ELR), and compare susceptibility percentages with those of the Centers for Disease Control and Prevention's Active Bacterial Core surveillance (ABCs) program.

\section{Introduction}

Since November 2014, the Houston Health Department has been receiving antimicrobial resistance information for Streptococcus pneumoniae from a safety net hospital via electronic laboratory reporting (ELR). Antimicrobial characteristics and vaccination rates of pneumococcal disease are of public health interest due to potential implications in treatment and prevention. Ten states participate in the CDC's Active Bacterial Core surveillance (ABCs) program. Texas, which represents a different and diverse demographic compared to other states, is not an ABCs participating state. No studies have compared local antimicrobial susceptibility percentages to those of the ABCs. The aim of this study is to 1) report the antimicrobial susceptibility of $S$. pneumoniae in a local cohort, 2) characterize the demographics of the cohort including the use of pneumococcal vaccine, and 3) compare antimicrobial susceptibility percentages of the local cohort to the $2013 \mathrm{ABCs}$ program.

\section{Methods}

Selected local antimicrobial susceptibility results received between November 2014 and July 2015 were compared to the ABCs susceptibilities using binomial methods. The data source for the local information is susceptibility ELRs submitted from the local safety net hospital lab, and the data source for the ABCs information is the 2013 ABCs report. ${ }^{1}$ The local study cohort consisted of individuals who had positive blood cultures and reported susceptibilities for $S$. pneumoniae at a safety net hospital in Houston $(\mathrm{N}=27)$. The susceptibility characterization of $S$. pneumoniae from ELR includes percentages susceptible, intermediate, and resistant by antimicrobial drug.

\section{Results}

From November 2014 to July 2015, there were 27 individuals with pneumococcal disease with reported susceptibilities. Of the 27 patients, $9(33 \%)$ had been vaccinated. The racial breakdown is $44 \%$ African American, 41\% Hispanic or Latino, and 15\% Caucasian. About 59\% were aged 50-64 years, while 26\% were aged 25-49 years at the time of disease onset. We found that erythromycin shares a similar percentage susceptible as its 2013 ABCs counterpart ( $p=0.206 ; 95 \%$ confidence interval 0.335 to 0.797 ), while penicillin has a significantly different percentage susceptible than the 2013 $\mathrm{ABCs}$ penicillin ( $\mathrm{p}<0.01 ; 95 \%$ confidence interval 0.224 to 0.612 ). The susceptibility profile of $S$. pneumoniae in the local hospital cohort is displayed in Table 1 below.

\section{Conclusions}

Statistical comparison of pneumococcal disease in a local safety net hospital cohort to ABCs statistics can identify differences in the susceptibility profile and use of pneumococcal vaccine. Erythromycin has a similar percentage susceptible in both cohorts, while penicillin has a significantly different percentage susceptible. The limitations of the study include the small sample size and potential biases due to multiple comparisons. The automated retrieval of susceptibility information via ELR is an important tool for public health surveillance. These findings, which are not population-based, can inform the design and development of enhanced population-based surveillance of pneumococcal disease based on ELR.

Table 1. Susceptibility profile of S. pneumoniae in a local hospital cohort, Houston, TX, November 2014 - July 2015

\begin{tabular}{|c|c|c|c|c|c|c|}
\hline & \multicolumn{2}{|c|}{ Susceptible } & \multicolumn{2}{c|}{ Intermediate } & \multicolumn{2}{c|}{ Resistant } \\
\hline Antimicrobial & $\mathrm{n}$ & $\%$ & $\mathrm{n}$ & $\%$ & $\mathrm{n}$ & $\%$ \\
\hline Penicillin & 11 & 39.3 & 15 & 53.6 & 2 & 7.1 \\
\hline Cefotaxime & 17 & 89.5 & 1 & 5.3 & 1 & 5.3 \\
\hline Erythromycin & 11 & 57.9 & - & - & 8 & 42.1 \\
\hline Levofloxacin & 19 & 100.0 & - & - & - & - \\
\hline Vancomycin & 8 & 100.0 & - & - & - & - \\
\hline
\end{tabular}

\section{Keywords}

electronic lab reporting; antimicrobial resistance; pneumococcal; Streptococcus pneumoniae; vaccination

\section{Acknowledgments}

The authors would like to thank Ryan Arnold and Nancy Vuong for their assistance in reviewing this manuscript.

\section{References}

1. Centers for Disease Control and Prevention. Active Bacterial Core Surveillance (ABCs) Report, Emerging Infections Program Network, Streptococcus pneumoniae, 2013 [Internet]. Centers for Disease Control and Prevention; 2015. Available from: http://www.cdc.gov/ abcs/reports-findings/survreports/spneu13.pdf

\section{*Avi Raju}

E-mail: Avi.Raju@houstontx.gov 